\title{
A New Approach to Blending and Loading Problem of Molten Aluminum
}

\author{
Li Jianhua \& Wei Xing \\ School of Mechanical \& Electronical Engineering, Lanzhou University of Technology, \\ 287 Langongping Rd, Qilihe, Lanzhou 730050, China \\ Email: li_jh@vip.sina.com
}

\begin{abstract}
The problems of blending electrolyzer and multi-constraint optimization of electrolytic aluminum scheduling in the electrolytic aluminum production process were addressed. Based on a mathematical model analysis, a novel hybrid optimization algorithm is proposed for optimization of blending together the molten aluminum in different electrolytic cells. An affinity degree function was designed to represent the path of aluminum scheduling. The mutation operators were designed to implement the transformation of electrolyzer combination and change the route of loading. A typical optimization example from an aluminum plant in northwest China is given in this paper, the results of which demonstrate the effectiveness of the proposed method.
\end{abstract}

Keywords: blending electrolyzer; chaos clone algorithm; distance of collecting path; electrolytic cell; process scheduling.

\section{$1 \quad$ Introduction}

Blending liquid aluminum together is a key operation process and is mainly done in an artificial way in the factory. The work is difficult and timeconsuming [1-3]. In allusion to the problems mentioned above, little research work has been conducted on this aspect [4]. Xia worked on algorithms for optimization of blending electrolytic cells without considering scheduling [5]. Denis Blanchard-Gaillard put forward heuristic solution approaches for assigning the output of electrolytic cells to oven batches in the production of aluminum, based on the levels of two impurities [6]. Optimization for the process of blending electrolytic cells with multi-constraint conditions pertaining to the aluminum ladle combined with two electrolytic cells was developed by Zhang Yali. Blending electrolytic cell scheduling has been optimized using the optimal objective combined with the $\mathrm{Fe}$ and $\mathrm{Si}$ content and the distance of the collecting path [7]. But in real production, blending electrolytic cells and scheduling is much more complex: the test data are complex and not only $\mathrm{Fe}$ and $\mathrm{Si}$ need to be computed; the electrolytic cell condition is much more complex as either the test data are so good that nothing needs to be done, or so bad that automatic blending and scheduling cannot be finished; the scheduling

Received April 25 ${ }^{\text {th }}, 2014$, Revised August $5^{\text {th }}, 2014$, Accepted for publication October $26^{\text {th }}, 2014$.

Copyright (C) 2014 Published by ITB Journal Publisher, ISSN: 2337-5779, DOI: 10.5614/j.eng.technol.sci.2014.46.4.8 
process is much more complex, the number of electrolytic cells used to form one aluminum ladle is variable, and there can be less than is acceptable.

Thus, in this paper a hybrid optimization algorithm, composed of an immune chaos clone algorithm and an artificial experience, is proposed for optimization of blending together the aluminum in different electrolytic cells and rule out special cases. The affinity degree function was optimized by the path of aluminum scheduling. The mutation operators were designed to implement the transformation of electrolyzer combination and change the route of loading. A typical optimization example from an aluminum plant in northwest China is given in this paper, the result of which demonstrates the effectiveness of the proposed method and indicates that the proposed algorithm produced better solutions than a genetic algorithm.

\section{Mathematical Modeling of Blending Electrolytic Aluminum Scheduling}

Blending liquid aluminum together means to extract the liquid aluminum from two or three electrolytic cells (Figure 1) into an aluminum ladle (Figure 2). In the production process, the assay results of every electrolytic cell, including ferrum $(\mathrm{Fe})$, silicon $(\mathrm{Si})$, magnesium $(\mathrm{Mg})$, cuprum $(\mathrm{Cu})$, calcium $(\mathrm{Ca})$ and zinc $(\mathrm{Zn})$, are different from each other due to using raw material. These chemicals decide the final purity of the aluminum. The impurity content of the blending liquid aluminum, including $\mathrm{Fe}, \mathrm{Si}, \mathrm{Mg}, \mathrm{Cu}, \mathrm{Ga}$ and $\mathrm{Zn}$, cannot exceed the production standard. The process of blending electrolytic cells is very complex in real production in the plant. The plan of blending electrolytic cells is not only closely related to the chemical composition of each electrolytic cell but also directed by the distance among the electrolytic cells that will be extracted into the same aluminum ladle (Figure 2). This distance is defined as the 'collecting path'.

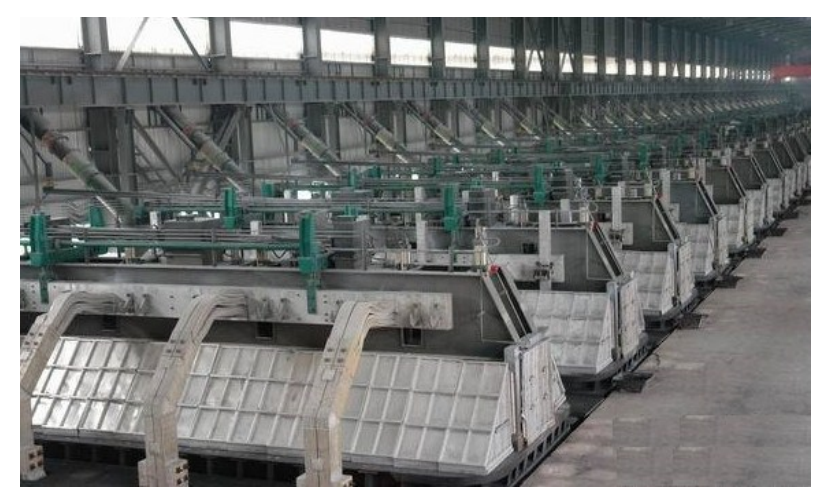

Figure 1 Electrolytic cells in an electrolytic aluminum plant. 


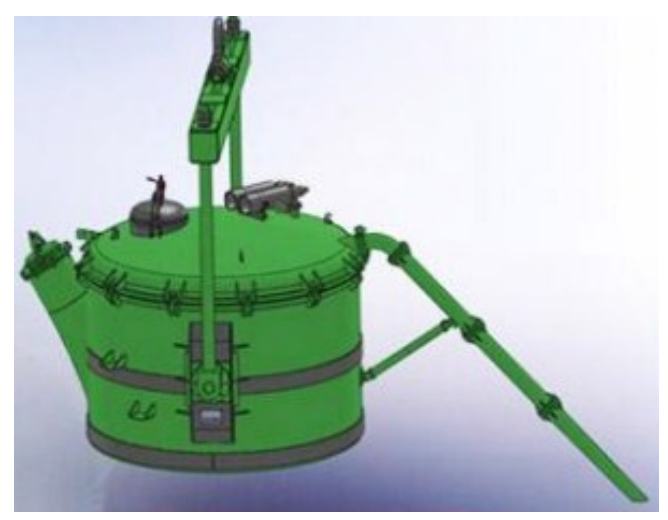

Figure 2 Aluminum ladle.

The electrolytic cells are kept in the same order, as shown in Figure 3. In production, each aluminum ladle is combined with two or three electrolytic cells. In the product standard testing premise, a plan for which three electrolytic cells are chosen to be combined, the aluminum ladle is worked out such that the purging path is the shortest possible.

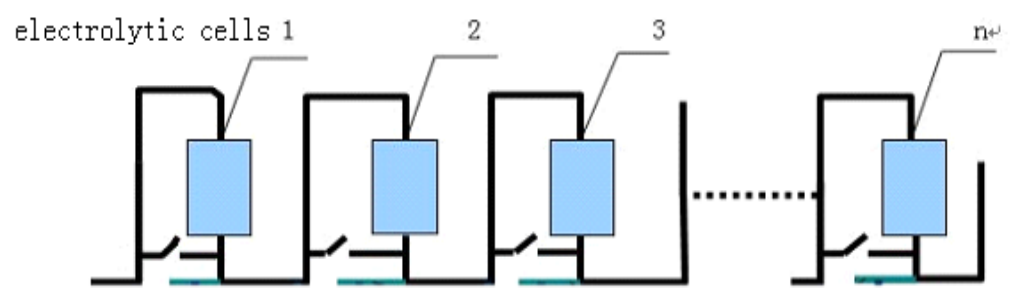

Figure 3 Arrangement of the electrolytic cells.

Definition 1. The set $N=\quad \ldots \quad \ldots \quad$ denotes the electrolytic cells.

Definition 2. The set $M_{1}, \ldots, M_{i}, \ldots M_{m}, M_{m+}$ denotes the maximum content of chemical impurity elements in the electrolytic cells, $m$ is the number of elements that the process requires to test for, $i \in \quad M_{i}$ denotes the element's maximum content, $M_{m+}$ denotes the maximum content for the sum of m elements.

Definition 3. The set $W_{1}, \ldots, W_{\mathrm{i}}, \ldots, W_{n}$ denotes the liquid aluminum weight that will be extracted into the aluminum ladle from the electrolytic cells at number $\mathrm{n}$. 
Definition 4. $P_{i 1}, \ldots, P_{i j}, \ldots P_{i m}, P_{i(\mathrm{~m}+}, P_{i j}$ denotes the content of the number $j$ element for the number $i$ electrolytic cell, $P_{i(\mathrm{~m}+}$ denotes the total content of all elements from number $i$ electrolytic cell.

Definition 5. $C_{i}=\quad c_{i 3}$ denotes the set of aluminum ladles, $c_{i 1}$, $c_{i 2}$ and $c_{i 3}$ are the number of electrolytic cells, $i$ is the number of the aluminum ladle. $0<i \leq\lfloor(n-1) / 3\rfloor+1$. We require $c_{i 1}<<$ in order to facilitate easy calculation (the number of electrolytic cells in the last aluminum ladle can be less than or equal to 3 ).

Definition 6. $L_{i}=\quad-\quad+\quad-\quad, L_{i}$ denotes the distance that the crown block drives for bringing liquid aluminum to aluminum ladle number $i$. If the last aluminum ladle only contains liquid aluminum from two electrolytic cells, the distance equals the distance between the two electrolytic cells. If the last aluminum ladle only contains liquid aluminum from one electrolytic cell, the distance equals 0 (the unit is the distance between two adjacent electrolytic cells).

Based on the blending process requirements, the shortest distance optimization function is defined as follows:

$$
L=\min \sum_{i=1}^{1+\lfloor(n-1) / 3\rfloor} L_{i} \quad i \in[1,1+\lfloor(n-1) / 3\rfloor]
$$

The computational constraints are:

$$
\sum_{k=1}^{s}\left(P_{c_{i k} j} \times W_{c_{i k}}\right) \leq M_{j} \times \sum_{k=1}^{s} W_{c_{i k}}
$$

where $\left\{\begin{array}{c}i \in[1,1+\lfloor(n-1) / 3\rfloor] \\ j \in[1, m+1] \\ 1 \leq s \leq 3\end{array}, s\right.$ denotes the number of electrolytic cells in

the current aluminum ladle.

\section{Model Solution Based on Hybrid Chaos Clone Algorithm}

According to the above discussion of blending and scheduling, there must be many constraints in the process of optimization during the computing process. It is very difficult to design an intelligent operator for a genetic algorithm because 
of the large amount of invalid solutions, such as the crossover operator in a genetic algorithm. A more simple and efficient operator needs to be put forward for the whole optimization process. Here, a novel hybrid optimization algorithm, composed of an immune chaos clone algorithm [8-9] and an artificial experience, is proposed.

\subsection{The Gene Code Rule and The Initialization}

The gene code is defined as $n_{1}, n_{2}, \ldots, n_{n}$, where $n_{i}$ is the number of the electrolytic cell. One aluminum ladle is formed by three electrolytic cells. For example, aluminum ladle 1 is composed of $n_{1}$ cell, $n_{2}$ cell, and $n_{3}$ cell. $n_{3(k-+}++\ldots+$ denotes an aluminum ladle group in the scheduling scheme. $k \in\left[1, \mathbb{4}\left(\begin{array}{l}n \\ )\end{array}\right]\right\rfloor$, when $k$ is the last group, the amount of electrolytic cells can be less than 3. For example, $(7,9,10,14,15,16,3,5,6,1,2,4,11,12,13,8)$ denotes that 16 electrolytic cells need to be blended, $(7,9,10)$ is the first aluminum ladle, $(14,15,16)$ is the second aluminum ladle, etc. The 6th aluminum ladle only contains one electrolytic cell (8).

During initialization we put emphasis on the content of Fe, as it is one of the most important indicators in production. The initialization can be described as follows:

Step 1. The initial position of electrolytic cell $i$ is produced between 1 and $n$. Then the sequential genetic chain $(i, i+1, i+2 \ldots . \mathrm{n}, 1,2 \ldots, i-1)$ is formed.

Step 2. According to the Fe content, from high to low, bubble sort is used to adjust the genetic chain.

Step 3. Starting at the first gene-bit, three bits in the genetic chain form one ladle from left to right. The gene-bits will be exchanged sequentially until the ladle ingredients satisfy the constraint condition in Formula 1.

Step 4. Repeat Step 3 until all gene-bits have found their ladle group. Finally, the initial genetic chain is formed.

\subsection{The Mutation Operator}

The mutation operator is used to implement the transformation of electrolytic cell combination and change the route of collecting. The mutation operator can be described as follows: 
Step 1. The mutation gene position $i$ is confirmed, guided by a chaotic vector [7], $3<<$. The $j$ position is set to 1 .

Step 2. Formula 1 is calculated after exchanging gene position $i$ and $j$. If $j=n$ or the result satisfies the inequality then the new exchanging gene chain is saved, the process jumps to the Step 4. else it progresses to the next step, $j=j+1$.

Step 3. This step forces $i 1=1+\lfloor(i-1) / 3\rfloor$ and $j 1=1+\lfloor(j-1) / 3\rfloor$ to assume integral values. If $i 1=j 1$, then $j=j+1$ and the process jumps to Step 3 . Else it jumps to Step 2.

Step 4. The program ends.

A mutation example can be shown as follows:

The original genetic chain : $(8,12,13,3,6,14,9,10,16,1,11,15,4,5,7,2)$

After mutation : $(8,12,13,3,6,14,9,10,16,1,11,2,4,5,7,15)$

\subsection{The Hybrid Optimization Algorithm}

Exceptions will be excluded before the optimizing calculation. In actual production, the test data of the liquid aluminum in the electrolytic cells are usually volatile. When the electrolytic cells' condition is good, so that all test data meet the requirements, nothing needs to be done but collect the liquid aluminum according to the nearby-cell principle. When the worst situation occurs, so that electrolytic cell blending cannot be executed due to the high content of some chemical component, the blending plan cannot be automatically computed but can only be dealt with manually. The two different conditions need to be excluded by the artificial experience.

The algorithm is as follows:

Step 1. Exclude the special case with the best condition. $c_{i k}$ is allocated a number from small to large in Definition 5. Formula 1 is calculated. The electrolytic cells' condition is to be excluded if all constraints are satisfied. Liquid aluminum will be directly collected according to the nearby-cell principle from small number to large number. The program ends.

Step 2. Exclude the special case with the worst condition. For any $N_{i} \in$, if there are not any two cells among the remaining cells that can satisfy all constraints of Formula 1, then the electrolytic cells' condition is bad. The blending plan cannot be automatically computed. The program ends. 
Step 3. Initial population. Population size is NS. The population is produced randomly and the parameters of the operators are set. The individual's fitness is calculated. Clone size is AS.

Step 4. Clonal expansion. A new population is generated by clone expansion of the individual according to AS. The new population size is $N S \times$.

Step 5. Chaotic clonal variation. The chaotic vector is calculated. If the value of the corresponding bit of the chaotic vector is 1 , this individual bit will be mutated. The individual's fitness is calculated after the mutation.

Step 6. Clonal selection operation. The individual with the best fitness in its clone expansion family is selected to be an individual in the younger generation. The younger generation size is reduced to NS.

Step 7. The terminal condition is examined. The process goes back to Step 4 only if the terminal condition is not satisfied.

Step 8. The best individual is selected.

\section{Implementation}

Test data from an aluminum plant in the northwest of China, where the purity of the aluminum product is required to reach $99.7 \%$, were used to validate our approach. The maximum impurity content allowed in this product is shown in Table 1; all of these constraints must be satisfied at the same time.

Table 1 Maximum content of chemical impurity elements (\%).

\begin{tabular}{lllllll}
\hline $\mathbf{F e}$ & $\mathbf{S i}$ & $\mathbf{C u}$ & $\mathbf{G a}$ & $\mathbf{M g}$ & $\mathbf{Z n}$ & Sum \\
\hline 0.2 & 0.1 & 0.01 & 0.03 & 0.02 & 0.03 & 0.3 \\
\hline
\end{tabular}

Sixteen electrolytic cells were selected from which to collect liquid aluminum. The test data of the liquid aluminum in the electrolytic cells are shown in Table 2.

The weight of one electrolytic cell is about three tons in actual production. We assigned three tons for each electrolytic cell's weight in order to make the calculation convenient and the result display intuitive. The initial population size was 10 and the clone size was 10 . The shortest distance of the collecting path for the initial population, which was randomly generated, was 48 . The optimal distance value was 14 after 20 generations, the collecting path was : $(1,2,3,14,15,16,4,5,6,9,10,11,7,8,13,12)$. 
Table 2 Test data of sixteen electrolytic cells (\%).

\begin{tabular}{cccccccc}
\hline $\begin{array}{c}\text { Cell } \\
\text { number }\end{array}$ & Fe & Si & $\mathbf{C u}$ & $\mathbf{G a}$ & $\mathbf{M g}$ & $\mathbf{Z n}$ & Sum \\
\cline { 1 - 6 } 1 & 0.21 & 0.05 & 0 & 0.02 & 0 & 0.01 & 0.29 \\
2 & 0.16 & 0.05 & 0 & 0.02 & 0 & 0.01 & 0.24 \\
3 & 0.14 & 0.06 & 0 & 0.02 & 0 & 0.01 & 0.23 \\
4 & 0.16 & 0.04 & 0 & 0.02 & 0 & 0.01 & 0.23 \\
5 & 0.22 & 0.04 & 0 & 0.02 & 0.01 & 0.01 & 0.3 \\
6 & 0.18 & 0.05 & 0 & 0.02 & 0 & 0.01 & 0.26 \\
7 & 0.16 & 0.04 & 0 & 0.02 & 0 & 0.01 & 0.23 \\
8 & 0.15 & 0.04 & 0 & 0.02 & 0 & 0.01 & 0.22 \\
9 & 0.17 & 0.06 & 0 & 0.02 & 0 & 0.01 & 0.26 \\
10 & 0.18 & 0.04 & 0 & 0.02 & 0 & 0.01 & 0.25 \\
11 & 0.18 & 0.04 & 0 & 0.02 & 0 & 0.01 & 0.25 \\
12 & 0.15 & 0.04 & 0 & 0.02 & 0 & 0.01 & 0.22 \\
13 & 0.28 & 0.04 & 0 & 0.02 & 0 & 0.01 & 0.35 \\
14 & 0.23 & 0.04 & 0 & 0.02 & 0 & 0.01 & 0.3 \\
15 & 0.18 & 0.05 & 0 & 0.02 & 0 & 0.01 & 0.26 \\
16 & 0.15 & 0.04 & 0 & 0.02 & 0 & 0.01 & 0.22 \\
\hline
\end{tabular}

Table 3 Bad test data of sixteen electrolytic cells (\%).

\begin{tabular}{cccccccc}
\hline $\begin{array}{c}\text { Cell } \\
\text { number }\end{array}$ & Fe & Si & $\mathbf{C u}$ & $\mathbf{G a}$ & $\mathbf{M g}$ & $\mathbf{Z n}$ & Sum \\
\cline { 1 - 5 } 1 & 0.16 & 0.05 & 0 & 0.02 & 0 & 0.01 & 0.24 \\
2 & 0.14 & 0.06 & 0 & 0.02 & 0 & 0.01 & 0.23 \\
3 & 0.16 & 0.04 & 0 & 0.02 & 0 & 0.01 & 0.23 \\
4 & 0.32 & 0.07 & 0 & 0.02 & 0 & 0.01 & 0.42 \\
5 & 0.15 & 0.04 & 0 & 0.02 & 0 & 0.01 & 0.22 \\
6 & 0.18 & 0.05 & 0 & 0.02 & 0 & 0.01 & 0.26 \\
7 & 0.16 & 0.04 & 0 & 0.02 & 0 & 0.01 & 0.23 \\
8 & 0.15 & 0.05 & 0 & 0.02 & 0 & 0.01 & 0.23 \\
9 & 0.17 & 0.05 & 0 & 0.02 & 0 & 0.01 & 0.25 \\
10 & 0.18 & 0.04 & 0 & 0.02 & 0 & 0.01 & 0.25 \\
11 & 0.18 & 0.04 & 0 & 0.02 & 0 & 0.01 & 0.25 \\
12 & 0.19 & 0.06 & 0 & 0.02 & 0 & 0.01 & 0.28 \\
13 & 0.15 & 0.06 & 0 & 0.02 & 0 & 0.01 & 0.24 \\
14 & 0.22 & 0.05 & 0 & 0.02 & 0 & 0.01 & 0.3 \\
15 & 0.18 & 0.04 & 0 & 0.02 & 0 & 0.01 & 0.25 \\
16 & 0.15 & 0.04 & 0 & 0.02 & 0 & 0.01 & 0.22 \\
\hline
\end{tabular}

In actual production, the test data of the liquid aluminum in the electrolytic cells are usually volatile. The bad test data and the good test data are shown in Table 3 and Table 4. The collecting path of the good test data was: $(1,2,3,4,5,6,7,8,9,10,11,12,13,14,15,16)$. The collecting path of the bad data could not be automatically computed. The algorithm noted that the test data were too bad to be dealt with manually. 
Table 4 Good test data of sixteen electrolytic cells (\%).

\begin{tabular}{cccccccc}
\hline Cell number & Fe & Si & Cu & Ga & Mg & Zn & Sum \\
\cline { 1 - 5 } 1 & 0.16 & 0.05 & 0 & 0.02 & 0 & 0.02 & 0.25 \\
2 & 0.18 & 0.05 & 0 & 0.02 & 0 & 0.01 & 0.26 \\
4 & 0.15 & 0.04 & 0 & 0.02 & 0 & 0.01 & 0.22 \\
5 & 0.16 & 0.05 & 0 & 0.02 & 0 & 0.03 & 0.26 \\
6 & 0.18 & 0.04 & 0 & 0.02 & 0 & 0.02 & 0.26 \\
7 & 0.15 & 0.05 & 0 & 0.02 & 0 & 0.03 & 0.25 \\
8 & 0.17 & 0.04 & 0 & 0.02 & 0 & 0.03 & 0.26 \\
9 & 0.19 & 0.04 & 0 & 0.02 & 0 & 0.01 & 0.26 \\
10 & 0.17 & 0.05 & 0 & 0.02 & 0 & 0.01 & 0.25 \\
11 & 0.15 & 0.04 & 0 & 0.02 & 0 & 0.01 & 0.22 \\
12 & 0.17 & 0.05 & 0 & 0.02 & 0 & 0.01 & 0.25 \\
13 & 0.17 & 0.05 & 0 & 0.02 & 0 & 0.01 & 0.25 \\
14 & 0.17 & 0.05 & 0 & 0.02 & 0 & 0.01 & 0.25 \\
15 & 0.16 & 0.05 & 0 & 0.02 & 0 & 0.01 & 0.24 \\
16 & 0.16 & 0.05 & 0 & 0.02 & 0 & 0.01 & 0.24 \\
\hline
\end{tabular}

In an earlier work, we have tried to complete this optimization using a genetic algorithm [10]. But GA's are relatively inefficient, as the design and calculation is very complex due to a lot of invalid solutions in the crossover process. In order to test the effectiveness of our approach, a comparative experiment between GA and our approach was set up to compute the test data of Table 1. The results of 20 computations with different parameters are shown in Table 5.

Table 5 The results of the experiment.

\begin{tabular}{cc|cccc}
\hline Algorithms & $\begin{array}{c}\text { Population } \\
\text { size }\end{array}$ & $\begin{array}{c}\text { Clone } \\
\text { size }\end{array}$ & $\begin{array}{c}\text { Evaluation } \\
\text { number }\end{array}$ & $\begin{array}{c}\text { Elapsed } \\
\text { time (s) }\end{array}$ & $\begin{array}{c}\text { Shortest distance } \\
\text { of collecting path }\end{array}$ \\
\hline $\begin{array}{c}\text { Genetic } \\
\text { algorithm }\end{array}$ & 100 & $/$ & 150 & 0.026618067 & 15.3 \\
$\begin{array}{c}\text { Hybrid chaos } \\
\text { clone algorithm }\end{array}$ & 10 & 10 & 20 & 0.000699 & 14.3 \\
\hline
\end{tabular}

\section{Conclusion}

The problems of blending electrolyzer and multi-constraint optimization of aluminum scheduling in the electrolytic aluminum production process were addressed in this paper. On the basis of establishing a mathematical model, a 
novel hybrid optimization strategy algorithm, where an artificial experience and a chaos clone algorithm were used to perform optimization, was put forward. The affinity degree function was optimized by the path of aluminum scheduling. The mutation operators were designed to implement the transformation of electrolyzer combination and change the route of loading. A typical optimization example from an aluminum plant in the northwest of China was given and the result demonstrated the effectiveness of the proposed method and indicated that the proposed algorithm produced better solutions than a genetic algorithm.

\section{References}

[1] Kumar, M. \& Rajotia, S., Integration of Scheduling with Computer Aided Process Planning, Journal of Materials Processing Technology, 138(1), pp. 297-300, 2003.

[2] Moon, C. \& Seo, Y., Evolutionary Algorithm for Advanced Process Planning and Scheduling in a Multi-plant, Computers \& Industrial Engineering, 48(2), pp. 311-325, 2005.

[3] Mencía, R., Sierra, M.R. \& Menciá, C., A Genetic Algorithm for JobShop Scheduling with Operators Enhanced by Weak Lamarckian Evolution and Search Space Narrowing, Natural Computing, 13(2), pp. 179-192, 2014.

[4] Shabtay, D., Gaspar, N. \& Kaspi, M., A Survey on Offline Scheduling with Rejection, Journal of Scheduling, 16(1), pp. 3-28, 2013.

[5] Xia, Y.M., Calculation Analysis and Software Development of Primary Aluminum Casting Optimized Proportioning Aluminum Method, Light Metals, 25(7), pp. 38-41, 2005.

[6] Denis, B-G., Yano, C.A. \& Leung, J.M.Y., Discrete Deterministic and Stochastic Blending Problems with Two Quality Characteristics: Aluminum Blending, IIE Transactions, 31(10), pp. 1001-1009, 1999.

[7] Zhang, Y.L. Research and Development of Arranging Package of Aluminum Electrolysis Cells Intelligently, PhD Dissertation, College of Electronical and Information Engineering, North China University of Technology, Beijing, China, 2009.

[8] Ronghua, S., A Novel Immune Clonal Algorithm for MO Problems, IEEE Transactions on Evolutionary Computation, 16(1), pp. 35-50, 2012.

[9] Golmakani, H.R. \& Namazi, A., An Artificial Immune Algorithm for Multiple-Route Job Shop Scheduling Problem, The International Journal of Advanced Manufacturing Technology, 63(1), pp. 77-86, 2012.

[10] Chryssolouris, G. \& Subramaniam, V., Dynamic Scheduling of Manufacturing Job Shops Using Genetic Algorithms, Journal of Intelligent Manufacturing, 12(3), pp. 281-293, 2001 